\title{
Star Electronics, Inc.: An Excel Based Case Using Financial Statement Analysis To Detect Fraud
}

Joseph M. Ragan, Saint Joseph's University

Andrew J. Hadley, Saint Joseph's University

Alexander P. Raymond, Saint Joseph's University

\begin{abstract}
The demand for forensic accounting within the corporate world is continually increasing. The services performed by forensic accountants help detect and prevent fraud within companies. This case is a study of forensic accounting that utilizes fraud methodology and detection techniques through a simulation engagement. Those who complete this case will proceed through a series of procedures that will lead to the formulation of potential fraudulent activity within the discussed company. The addressed procedures include: understanding the business, determining the areas that may be susceptible to fraud, performing analytical procedures to detect fraud, and creating a report to the board of directors as a follow up to any potential red flags found during the performed testing.
\end{abstract}

\section{INTRODUCTION}

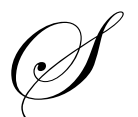

tar Electronics, Inc., a large national electronics retailer, has been trying to acquire its largest competitor, United Electronics. Star Electronics' board of directors requires that the books of United Electronics be audited before the acquisition is authorized. The board of directors has reason to believe potential unethical business decisions are being made by United Electronics' management. As a result, you and your team of forensic accountants have been asked to perform a comprehensive analysis of the financial statements, along with due diligence with regards to the structure and operations of management.

\section{THE TARGET COMPANY}

United Electronics is an electronics company that produces high end audio/video equipment. The company was formed in a small southern town. The three founders have previously worked together for a small electronics retailer, and had little experience with the production aspect of the audio/video industry. Robert Hadley became the chairman and chief executive officer, and Luke Raymond became the company's president and chief operating officer. The third person, Chris Reagan, became the controller and treasurer. Two of the three founders own, together, over $21 \%$ of the company's stock. The board of directors had a total of seven members, all of whom were related to, or close friends with, the founders. Excited about the new company, the officers were always excited about the prospects of their start-up company.

United Electronics is an entity that produced a net income of $\$ 49$ million last year. There average accounts payable at year-end for the past two years were around $\$ 6$ million. The organizational structure, in terms of payroll, is segmented into two divisions, salary and hourly employees. Salary employees are administrative positions responsible for the non-production activities of the business, and, on the other hand, the hourly employees make up the production personnel within United. The company operates one major productions facility, making various electronic products for sale at major retail locations. 


\section{INTERNAL CONTROL ENVIRONMENT}

The two primary owners of the business, Hadley and Raymond, take a pretty hands-off approach when it comes to managing their business. They, and their families, take frequent vacations to various destinations in Europe, and rarely take the time to examine the business' books. They are most happy to leave Chris run the show, noting that there is little, if anything, to worry about as the bottom line steadily increases year after year. Reagan is very much hands-on in all aspects of the business. As he is always busy, Reagan is constantly concerned about the efficiency of the accounting system. In order to facilitate quicker purchases and obtain deals with vendors, Chris left his cashier a stack of blank, pre-signed checks. Anyone in the purchasing department has access to these checks, and may use them as needed. When it comes to payroll, United uses a one-write system to pay its employees, and checks are distributed to the employee mailing addresses at the end of every two weeks. In order to manage internal controls within the sales cycle transactions, Chris personally approves all credit given to customers. Chris insists that it is best for one person to be in charge.

\section{INVESTMENT DECISION}

The acquisition of United would give Star Electronics the number one position within their industry. What attracted Star to United was the remarkable growth of the company, and the issuance of a major recommendation to buy from a large brokerage firm. The recommendation was specifically based on 35\% earnings per share growth, and comparable product line growth in the low double digit range over the past two years. As a result of the acquisition, Star Electronics would strengthen their relationships with existing vendors, and expand their already extensive customer base. In attempting to negotiate a reasonable purchase price, Star Electronics has asked its analysts to review financial statements prior to negotiation. Star asked the analyst to ensure that United's assumptions were reasonable and realistically attainable given the current market for consumer electronics.

\section{THE TEAM CHALLENGE}

Star Electronics would like to hire you and your team to conduct a financial statement review for the past two years of their operations. Although fraud is unlikely, your detection efforts should be thorough. Here is some guidance for the analytical procedures that should be performed:

- Unusual sales increase, especially at the end of each quarter.

- $\quad$ Large amount of receivables that have aged over sixty days.

- $\quad$ Sharp decreases in accrued expenses, particularly at the end of the quarters.

- $\quad$ Sudden shifts in unearned revenue accounts.

- Due to their weak internal control environment, United's vendors and customers should be thoroughly checked for validity and existence.

- $\quad$ A search for vendors with unusual names and sequential invoices.

- $\quad$ Trend analysis of revenue flow should be performed.

- $\quad$ Thorough review of payroll records provided for all salary employees should be conducted.

Financial statements are the end product of the accounting cycle. They can be viewed as summaries of all transactions that occur within a company during a period of time. Financial statement fraud can occur anywhere along the way. Many large frauds are detected through financial statement comparisons. Smaller frauds are usually detected by focusing on individual ledgers and source documents.

At the conclusion of your analytical procedures, you and your team should compile a comprehensive report to the board of directors that displays any potential fraudulent activity that was found. This information will be used by the board of directors to determine if further investigation and/or legal action needs to be taken. This analysis conducted by you and your team is extremely important to the outcome of this pending acquisition. 


\section{TEACHING NOTES}

\section{Synopsis}

This case whas been created as a means to further student's overall understanding of fraudulent activities within a company through means of hands on investigation. Students receive two years of financial statements, along with a payroll register, and schedules of Accounts Receivable and Acounts Payable. They are asked to examine each of these documents thoroughly and search for potential red flags. In addition to finding red flags, students are required to create a detailed report on their findings, simulating a real life investigation. Some of the issues that occur are specific to each document, while there are others that require students to look at the bigger picture. An answer guide is included for the instructor, along with a set of journal entries and a graph that may be given to the students after completing the case to promote further discussion. Selected References have been incorporated into the teaching notes as a basis for the type of course in which this case would fit. The case has been successfully utilized in a forensic accounting special topics graduate accounting course and in an undergraduate forensic accounting course. In both cases, students worked in groups of three or less.

\section{Analysis}

The analysis of this case can be broken down into 4 parts. These parts include:

1. Analysis of the Balance Sheet and Income Statement

2. Analysis of Accounts Receivable

3. Analysis of Accounts Payable

4. Analysis of Payroll Register investigation.

In each of these areas, there are multiple issues that will focus on several concepts of a forensic

In the Balance Sheet and Income Statement the following issues are included:

1. Increasing Sales with no increase in Inventory or Cost of Goods Sold

2. Artificial Increasing in Sales Revenue by decreasing Accrued Expenses

3. An increase in Assets offset by a decrease in costs

4. A decrease in Unearned Revenues, specifically at the end of quarters, to help inflate Sales

5. A graph is included to shows a trend in Sales increase that may be used in further discussion

In Accounts Receivable the following issues are included:

1. There is an issue with the total value of $\mathrm{A} / \mathrm{R}$ not tying to the Balance Sheet. This relates to the Balance Sheet and Income Statement matters listed above, and a set of journal entries is included for use by the instructor in providing further clarification to students about the entries used in creating fraudulent transactions.

2. There are a large number of Receivables in the "over 90 days" category

In Accounts Payable the following issues are included:

1. A company having two invoices with identical invoice numbers, falling in two different aging categories.

2. Companies having the same addresses as employees.

In the Payroll Register the following issues are included:

1. Multiple Employees with the same address

2. Multiple Employees with the same Social Security Number 
3. Employees with slightly different names having the same PO Box

4. $\quad$ Employees whose ages would make them too young to legally work

5. Employees with odd names that could be fictitious

\section{Answer Guide}

The answer guide has been created as a means for instructors to see the key points of concern within the financial statements. Students may not be required to find each specific potential fraud, and likewise it is possible that other issues may exist other than those which are noted.

\section{Inventory}

-Although Sales Increase in 2006 (especially in the end of the quarters), Inventory and Cost of Goods Sold remain fairly constant. December 2006 is an example of this.

\section{Accrued Expenses}

-Accrued Expenses decreased sharply starting in the $2^{\text {nd }}$ quarter of 2006 in order to artificially inflate sales (Please see attached Journal Entries).

\section{Other Current Assets}

-In December of 2006, Other Current Assets increase. There is a corresponding decrease in Engineering and Marketing Costs.

\section{Unearned Revenue}

-Balances decrease at the end of the first and second quarter of 2006. Amounts were reversed to help inflate sales (See attached Journal Entries).

\section{Accounts Receivable}

-There are a large number of "Over 90 days" Receivables.

-The totals of A/R do not tie to the Financial's totals of A/R for 2006 . They are off by $\$ 11$ million, the amount of two "topside" journal entries to Bob Smith, used to inflate sales. (See attached Journal Entries)

\section{Accounts Payable}

-The payables for IDX have two invoices with the same number in different aging buckets.

-Nelson's Consulting and Nelson's Temp Agency have the same address as the Nelson employees (see Payroll Register section for the issues pertaining to the Nelson employees).

-Y Ask For Anything More's invoice numbers are out of sequence compared to the aging schedule.

-John's Electric Surplus and Timmy's Electronic Shop have the same address and the same invoiced dollar amounts. -Cam's Exotic Cars- Why is the company spending money at an exotic car dealership?

\section{Payroll Register}

-Stealing, I.M. is a curious name that should draw a red flag.

-Hollywood, L. is too young to work as he was born in 1997. Also his date of employment is in 1990, seven years before his birth.

-Hafer, F., and Szurgot, Z. have the exact same address.

-Osmond, E., and Weicicoskie, C. have the same Social Security Number.

- Nelson, M., Nellson, M., and Nelse, M. have very similar names and have an Identical PO Box.

This PO Box is also shared with Nelson's Consulting and Nelson's Temp Agency on the Accounts Payable Aging Report. 


\section{Sales}

-Sales were artificially inflated at the end of each quarter of 2006 using reversals of Accrued Expenses and Unearned Revenues, as well as through inflation of Accounts Receivable. (See attached Journal Entries)

-By graphing monthly sales, a clear trend is created showing spurratic sales increases at the end of each quarter (Please see attached graph).

\section{Journal Entries}

\begin{tabular}{|c|c|c|c|}
\hline 1st Quarter & Unearned Revenue & $1,750,000$ & \\
\hline & Sales & & $1,750,000$ \\
\hline \multirow[t]{2}{*}{ 2nd Quarter } & Unearned Revenue & $3,100,000$ & \\
\hline & Sales & & $3,100,000$ \\
\hline \multirow[t]{2}{*}{ 2nd Quarter } & Accrued Expenses & $1,500,000$ & \\
\hline & Sales & & $1,500,000$ \\
\hline \multirow[t]{2}{*}{ 3rd Quarter } & Accrued Expenses & 900,000 & \\
\hline & Sales & & 900,000 \\
\hline \multirow[t]{2}{*}{ 3rd Quarter } & A/R- Bob Smith & $3,000,000$ & \\
\hline & Sales & & $3,000,000$ \\
\hline \multirow[t]{3}{*}{ 3rd Quarter } & Other Current Assets & 365,000 & \\
\hline & Engineering & & 161,097 \\
\hline & Marketing & & 203,904 \\
\hline \multirow[t]{2}{*}{ 4th Quarter } & A/R- Bob Smith & $8,000,000$ & \\
\hline & Sales & & $8,000,000$ \\
\hline \multirow[t]{3}{*}{ 4th Quarter } & Other Current Assets & 380,000 & \\
\hline & Engineering & & 168,597 \\
\hline & Marketing & & 211,404 \\
\hline
\end{tabular}




\section{Monthly Sales Graph}

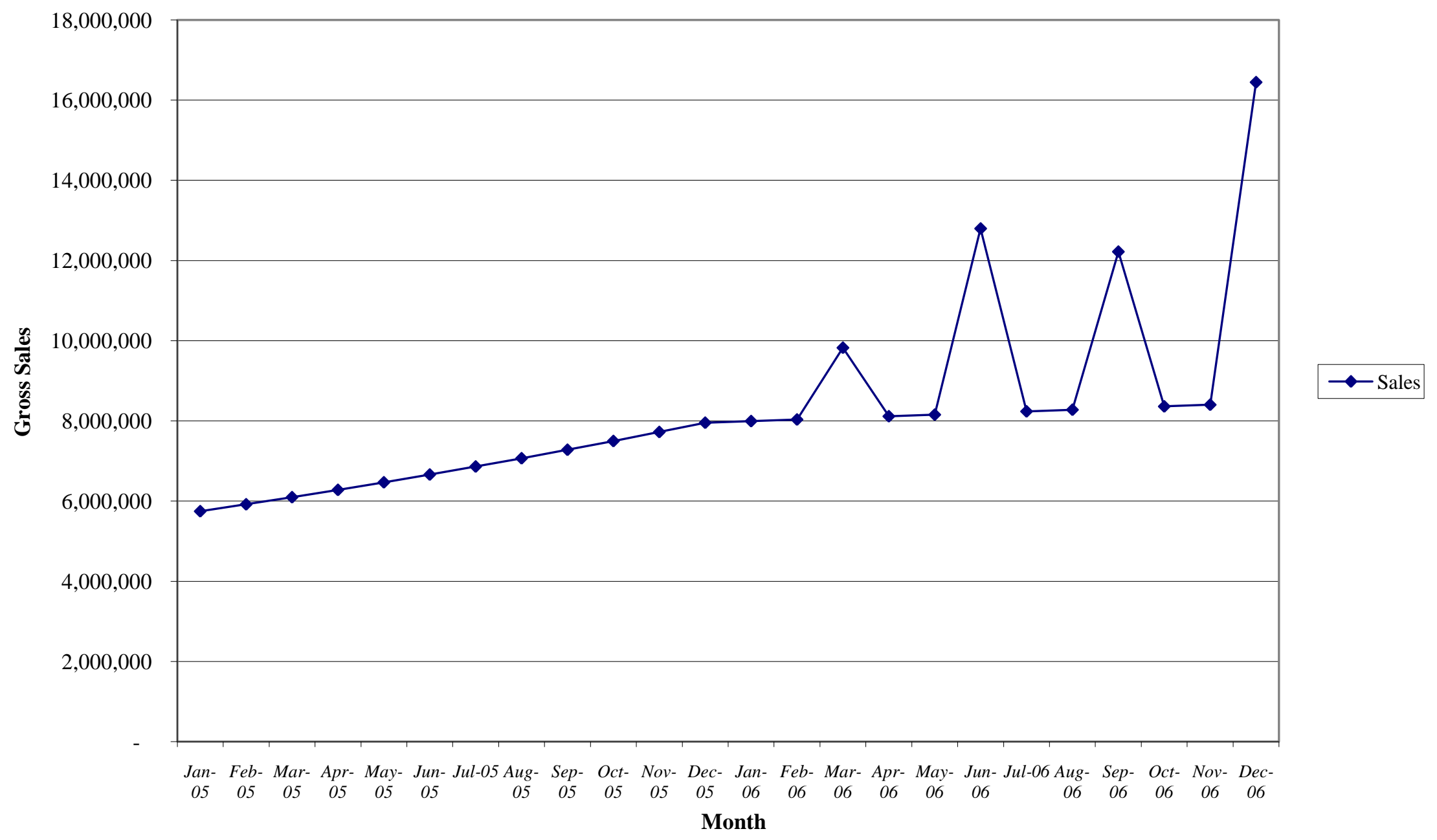




\section{SELECTED REFERENCES}

1. $\quad$ Albrecht, W. Steve. Fraud Examination. Mason, Ohio, Thomson South-Western, 2003.

2. Albrecht, W. Steve, Keith R. Howe, and Marshall B. Romney. Deterring Fraud: The Internal Auditor's Perspective. Altamonte Springs: The Institute of Internal Auditors Research Foundation, 1984.

3. Albrecht, W. Steve, Gerald W. Wernz, and Timothy L. Williams. Fraud: Bringing Light to the Dark Side of Business. Burr Ridge, IL: Irwin Professional Publishing, 1995.

4. Albrecht, W. Steve, Marshal B. Romney, David J. Cherrington, I. Reed Payne, and Allan J. Roe. How to Detect and Prevent Business Fraud. Englewood Cliffs: Prentice-Hall, Inc., 1982.

5. American Institute of Certified Public Accountants, Inc. Accounting Standards, Original Pronouncements.

- The Auditor's Responsibility to Detect and Report Errors and Irregularities, SAS No. 53. New York, NY, 1990.

- $\quad$ Illegal Acts by Clients, SAS No. 54. New York, NY, 1990.

- $\quad$ Auditing Accounting Estimates, SAS No. 57. New York, NY, 1990.

- $\quad$ Communication with Audit Committees, SAS No. 61. New York, NY, 1990.

- Special Reports, SAS No. 62

- Compliance Auditing Applicable to Governmental Entities and Other Recipients

of Governmental Financial Assistance, SAS No. 63.

- $\quad$ Consideration of Fraud in a Financial Statement Audit, SAS No. 82, 1996.

6. Androphy, Joel M. White Collar Crime. New York: McGraw-Hill, Inc., 1992.

7. Arens, Alvin A., Randal J. Elder, and Mark S. Beasley. Essential Auditing and Assurance Services: An Integrated Approach. Upper Saddle River, New Jersey, Prentice Hall, 2003.

8. Association of Certified Fraud Examiners, The. 2004 Report to the Nation: Occupational Fraud and Abuse. ACFE, 2004.

9. Association of Certified Fraud Examiners, The. Fraud Examiners' Manual, Revised $4^{\text {th }}$ Edition. ACFE, 2003.

10. Bintliff, Russell L. White Collar Crime Detection and Prevention. Englewood Cliffs, New Jersey: Prentice Hall, 1993.

11. Bologna, Jack and Robert J. Lindquist. Fraud Auditing and Forensic Accounting. New York: John Wiley \& Sons, 1987.

12. Cressey, Donald R. Other People's Money. Montclair: Patterson Smith, 1953.

13. Davia, Howard R., Patrick C. Coggins, John C. Wideman, and Joseph T. Kastantin. Accountant's Guide to Fraud Detection and Control, Second Edition. New York, John Wiley \& Sons, 2000.

14. Drake, John D. The Effective Interviewer: A Guide for Managers. New York: AMACOM, 1989.

15. Fusaro, Peter C. and Ross M. Miller. What Went Wrong at Enron. Hoboken: John Wiley \& Sons, Inc., 2002.

16. Fridson, Martin S. Financial Statement Analysis. New York: John Wiley \& Sons, Inc., 1991.

17. Geis, Gilbert. On White-Collar Crime. Lexington, Mass: Lexington Books, 1982.

18. Inbau, Fred E., John E. Reid, and Joseph P. Buckley. Criminal Interrogation and Confessions. Baltimore: Wilkins, 1986.

19. Ketz, J. Edward. Hidden Financial Risks: Understanding Off-Balance Sheet Accounting. Hoboken: John Wiley \& Sons, 2003.

20. Koletar, Joseph W. Fraud Exposed: What You Don't Know Could Cost Your Company Millions. Hoboken: John Wiley \& Sons, 2003.

21. Lundelius Jr., Charles R. Financial Reporting Fraud: A Practical Guide to Detection and Internal Control. New York: AICPA, Inc. 2003.

22. Marcella, Albert J., William J. Sampias and James K. Kincaid. The Hunt for Fraud: Prevention and Detection Techniques. Altamonte Springs: Institute of Internal Auditors, 1994.

23. Rabon, Don. Investigative Discourse Analysis. Durham: Carolina Academic Press, 1994.

24. Seidler, Lee J., Fredrick Andrews, and Marc J. Epstein. The Equity Funding Papers: the Anatomy of a Fraud. New York: John Wiley \& Sons, 1997. 
25. Silverstone, Howard and Michael Sheetz. Forensic Accounting and Fraud Investigation for Non-Experts. Hoboken, John Wiley \& Sons, 2004.

26. Snyder, Neil H., O. Whitfield, William J. Kehoe, James T. McIntyre, Jr., and Karen E. Blair. Reducing Employee Theft: A Guide to Financial and Organizational Controls. New York: Quorum Books, 1991.

27. Thornhill, William T. Forensic Accounting: How to Investigate Financial Fraud. Burr Ridge, IL: Irwin Professional Publishing, 1995.

28. Tyler, Tom R. Why People Obey the Law. New Haven: Yale University Press, 1990.

29. Wells, Joseph T., CFE, CPA. The Encyclopedia of Fraud. Austin, Texas: Obsidian Publishing Company, Inc.; 2002.

30. Wells, Joseph T., CFE, CPA. Fraud Examination: Investigative and Audit Procedures. New York: Quorum Books, 1992.

31. Wells, Joseph T., CFE, CPA. Occupational Fraud and Abuse. Austin, Texas: Obsidian Publishing Company, Inc, 1997.

32. Zack, Gerard M. Fraud and Abuse in Nonprofit Organizations: A Guide to Prevention and Detection. Hoboken, John Wiley \& Sons, 2003. 


\begin{tabular}{|c|c|c|c|c|c|c|c|c|c|c|c|c|c|}
\hline 2005 Income Statement & $12 / 31 / 05$ & Dec-05 & Nov-05 & Oct-05 & Sep-05 & Aug-05 & Jul-05 & Jun-05 & May-05 & Apr-05 & Mar-05 & Feb-05 & Jan-05 \\
\hline SALES & Consolidated & & & & & & & & & & & & \\
\hline Gross Sales & $81,533,210$ & $7,952,424$ & $7,720,800$ & $7,495,922$ & $7,277,594$ & $7,065,625$ & $6,859,830$ & $6,660,030$ & $6,466,048$ & $6,277,717$ & $6,094,871$ & $5,917,350$ & $5,745,000$ \\
\hline Discounts & $(2,445,996)$ & $(238,573)$ & $(231,624)$ & $(224,878)$ & $(218,328)$ & $(211,969)$ & $(205,795)$ & $(199,801)$ & $(193,981)$ & $(188,331)$ & $(182,846)$ & $(177,521)$ & $(172,350)$ \\
\hline Returns \& Allowances & $(1,630,664)$ & $(159,048)$ & $(154,416)$ & $(149,918)$ & $(145,552)$ & $(141,313)$ & $(137,197)$ & $(133,201)$ & $(129,321)$ & $(125,554)$ & $(121,897)$ & $(118,347)$ & $(114,900)$ \\
\hline NET SALES & $77,456,549$ & $7,554,802$ & $7,334,760$ & $7,121,126$ & $6,913,714$ & $6,712,344$ & $6,516,839$ & $6,327,028$ & $6,142,746$ & $5,963,831$ & $5,790,127$ & $5,621,483$ & $5,457,750$ \\
\hline \multicolumn{14}{|l|}{ COST OF SALES } \\
\hline Standard Cost of Sales & $12,229,981$ & $1,192,864$ & $1,158,120$ & $1,124,388$ & $1,091,639$ & $1,059,844$ & $1,028,975$ & 999,004 & 969,907 & 941,657 & 914,231 & 887,603 & 861,750 \\
\hline Manufacturing Variances & 407,666 & 39,762 & 38,604 & 37,480 & 36,388 & 35,328 & 34,299 & 33,300 & 32,330 & 31,389 & 30,474 & 29,587 & 28,725 \\
\hline Total Cost of Sales & $12,637,648$ & $1,232,626$ & $1,196,724$ & $1,161,868$ & $1,128,027$ & $1,095,172$ & $1,063,274$ & $1,032,305$ & $1,002,237$ & 973,046 & 944,705 & 917,189 & 890,475 \\
\hline GROSS MANUFAC PROFIT & $64,818,902$ & $6,322,177$ & $6,138,036$ & $5,959,258$ & $5,785,687$ & $5,617,172$ & $5,453,565$ & $5,294,723$ & $5,140,508$ & $4,990,785$ & $4,845,422$ & $4,704,293$ & $4,567,275$ \\
\hline \multicolumn{14}{|l|}{ OPERATING EXPENSES } \\
\hline Engineering & $1,630,664$ & 159,048 & 154,416 & 149,918 & 145,552 & 141,313 & 137,197 & 133,201 & 129,321 & 125,554 & 121,897 & 118,347 & 114,900 \\
\hline Marketing & $2,038,330$ & 198,811 & 193,020 & 187,398 & 181,940 & 176,641 & 171,496 & 166,501 & 161,651 & 156,943 & 152,372 & 147,934 & 143,625 \\
\hline Salary Expenses & $6,127,500$ & 617,500 & 617,500 & 617,500 & 475,000 & 475,000 & 475,000 & 475,000 & 475,000 & 475,000 & 475,000 & 475,000 & 475,000 \\
\hline Depreciation Expense & 601,032 & 61,578 & 59,210 & 56,932 & 54,743 & 52,637 & 50,613 & 48,666 & 46,794 & 44,995 & 43,264 & 41,600 & 40,000 \\
\hline Total Operating Expenses & $10,397,527$ & $1,036,937$ & $1,024,146$ & $1,011,749$ & 857,234 & 845,590 & 834,305 & 823,367 & 812,767 & 802,492 & 792,533 & 782,881 & 773,525 \\
\hline OPERATING PROFIT & $54,421,375$ & $5,285,240$ & $5,113,890$ & $4,947,509$ & $4,928,453$ & $4,771,582$ & $4,619,260$ & $4,471,356$ & $4,327,742$ & $4,188,293$ & $4,052,889$ & $3,921,413$ & $3,793,750$ \\
\hline \multicolumn{14}{|l|}{ NON-OPERATING INCOME } \\
\hline Interest Expense & $(163,066)$ & $(15,905)$ & $(15,442)$ & $(14,992)$ & $(14,555)$ & $(14,131)$ & $(13,720)$ & $(13,320)$ & $(12,932)$ & $(12,555)$ & $(12,190)$ & $(11,835)$ & $(11,490)$ \\
\hline Other & $(40,767)$ & $(3,976)$ & $(3,860)$ & $(3,748)$ & $(3,639)$ & $(3,533)$ & $(3,430)$ & $(3,330)$ & $(3,233)$ & $(3,139)$ & $(3,047)$ & $(2,959)$ & $(2,873)$ \\
\hline Total Non-Operating Inc/Exp & $(203,833)$ & $(493,797)$ & $(493,797)$ & $(493,797)$ & $(493,797)$ & $(493,797)$ & $(493,797)$ & $(493,797)$ & $(493,797)$ & $(493,797)$ & $(493,797)$ & $(493,797)$ & $(493,797)$ \\
\hline PRETAX INCOME & $54,217,542$ & $4,791,442$ & $4,620,093$ & $4,453,712$ & $4,434,656$ & $4,277,785$ & $4,125,463$ & $3,977,559$ & $3,833,945$ & $3,694,496$ & $3,559,092$ & $3,427,615$ & $3,299,953$ \\
\hline PROVISION FOR INCOME TAXES & $21,144,841$ & $1,868,663$ & $1,801,836$ & $1,736,948$ & $1,729,516$ & $1,668,336$ & $1,608,931$ & $1,551,248$ & $1,495,238$ & $1,440,853$ & $1,388,046$ & $1,336,770$ & $1,286,982$ \\
\hline NET INCOME & $33,072,701$ & $2,922,780$ & $2,818,257$ & $2,716,764$ & $2,705,140$ & $2,609,449$ & $2,516,532$ & $2,426,311$ & $2,338,706$ & $2,253,642$ & $2,171,046$ & $2,090,845$ & $2,012,971$ \\
\hline
\end{tabular}




\begin{tabular}{|c|c|c|c|c|c|c|c|c|c|c|c|c|c|}
\hline \multirow[t]{2}{*}{2005 Balance Sheet } & $12 / 31 / 05$ & Dec-05 & Nov-05 & Oct-05 & Sep-05 & Aug-05 & Jul-05 & Jun-05 & May-05 & Apr-05 & Mar-05 & Feb-05 & Jan-05 \\
\hline & \multicolumn{13}{|l|}{$\underline{\text { Consolidated }}$} \\
\hline CASH AND ST INVESTMENTS & $10,000,000$ & $10,000,000$ & $10,000,000$ & $10,000,000$ & $10,000,000$ & $10,000,000$ & $10,000,000$ & $10,000,000$ & $10,000,000$ & $10,000,000$ & $10,000,000$ & $10,000,000$ & $10,000,000$ \\
\hline \multicolumn{14}{|l|}{ ACCOUNTS RECEIVABLE } \\
\hline Gross Accounts Receivable & $1,590,485$ & $1,590,485$ & $1,544,160$ & $1,499,184$ & $1,455,519$ & $1,413,125$ & $1,371,966$ & $1,332,006$ & $1,293,210$ & $1,255,543$ & $1,218,974$ & $1,183,470$ & $1,149,000$ \\
\hline Reserve for Doubtful Accounts & $(159,048)$ & $(159,048)$ & $(154,416)$ & $(149,918)$ & $(145,552)$ & $(141,313)$ & $(137,197)$ & $(133,201)$ & $(129,321)$ & $(125,554)$ & $(121,897)$ & $(118,347)$ & $(114,900)$ \\
\hline Net Accounts Receivable & $1,431,436$ & $1,431,436$ & $1,389,744$ & $1,349,266$ & $1,309,967$ & $1,271,813$ & $1,234,769$ & $1,198,805$ & $1,163,889$ & $1,129,989$ & $1,097,077$ & $1,065,123$ & $1,034,100$ \\
\hline INVENTORIES & $6,992,071$ & $6,992,071$ & $7,208,321$ & $7,431,259$ & $7,661,091$ & $7,898,032$ & $8,142,301$ & $8,394,125$ & $8,653,737$ & $8,921,379$ & $9,197,298$ & $9,481,750$ & $9,775,000$ \\
\hline OTHER CURRENT ASSETS & $2,300,000$ & $2,300,000$ & $2,300,000$ & $2,300,000$ & $2,300,000$ & $2,300,000$ & $2,300,000$ & $2,300,000$ & $2,300,000$ & $2,300,000$ & $2,300,000$ & $2,300,000$ & $2,300,000$ \\
\hline TOTAL CURRENT ASSETS & $20,723,507$ & $20,723,507$ & $20,898,065$ & $21,080,525$ & $21,271,058$ & $21,469,845$ & $21,677,071$ & $21,892,930$ & $22,117,626$ & $22,351,368$ & $22,594,374$ & $22,846,873$ & $23,109,100$ \\
\hline \multicolumn{14}{|l|}{ FIXED ASSETS } \\
\hline Land and Buildings & $8,966,667$ & $8,966,667$ & $8,983,334$ & $9,000,001$ & $9,016,668$ & $9,033,335$ & $9,050,002$ & $9,066,669$ & $9,083,336$ & $9,100,003$ & $9,116,670$ & $9,133,337$ & $9,150,004$ \\
\hline Machinery and Equipment & $1,558,333$ & $1,558,333$ & $1,566,666$ & $1,574,999$ & $1,583,332$ & $1,591,665$ & $1,599,998$ & $1,608,331$ & $1,616,664$ & $1,624,997$ & $1,633,330$ & $1,641,663$ & $1,649,996$ \\
\hline \multicolumn{14}{|l|}{ Construction in Progress } \\
\hline Accumulated Depreciation & 976,032 & 976,032 & 909,454 & 845,244 & 783,312 & 723,569 & 665,932 & 610,319 & 556,653 & 504,859 & 459,864 & 416,600 & 375,000 \\
\hline Net Fixed Assets & $11,501,032$ & $11,501,032$ & $11,459,454$ & $11,420,244$ & $11,383,312$ & $11,348,569$ & $11,315,932$ & $11,285,319$ & $11,256,653$ & $11,229,859$ & $11,209,864$ & $11,191,600$ & $11,175,000$ \\
\hline OTHER ASSETS & 875,000 & 875,000 & 875,000 & 875,000 & 875,000 & 875,000 & 875,000 & 875,000 & 875,000 & 875,000 & 875,000 & 875,000 & 875,000 \\
\hline TOTAL ASSETS & $33,099,540$ & $33,099,540$ & $33,232,519$ & $33,375,769$ & $33,529,370$ & $33,693,414$ & $33,868,003$ & $34,053,249$ & $34,249,279$ & $34,456,226$ & $34,679,238$ & $34,913,473$ & $35,159,100$ \\
\hline \multicolumn{14}{|l|}{ CURRENT LIABILITIES } \\
\hline Accounts Payable & $5,752,500$ & $5,752,500$ & $5,608,688$ & $5,468,470$ & $5,331,759$ & $5,198,465$ & $5,068,503$ & $4,941,790$ & $4,818,246$ & $4,697,790$ & $4,580,345$ & $4,465,836$ & $4,354,190$ \\
\hline Accrued Expenses & $1,900,000$ & $1,900,000$ & $1,900,000$ & $1,900,000$ & $1,900,000$ & $1,900,000$ & $1,900,000$ & $1,900,000$ & $1,900,000$ & $1,900,000$ & $1,900,000$ & $1,900,000$ & $1,900,000$ \\
\hline Unearned Revenue & $4,771,454$ & $4,771,454$ & $4,632,480$ & $4,497,553$ & $4,366,556$ & $4,239,375$ & $4,115,898$ & $3,996,018$ & $3,879,629$ & $3,766,630$ & $3,656,922$ & $3,550,410$ & $3,447,000$ \\
\hline Accrued Compensation & $2,200,000$ & $2,200,000$ & $2,200,000$ & $2,200,000$ & $2,200,000$ & $2,200,000$ & $2,200,000$ & $2,200,000$ & $2,200,000$ & $2,200,000$ & $2,200,000$ & $2,200,000$ & $2,200,000$ \\
\hline TOTAL CURRENT LIABILITIES & $14,623,954$ & $14,623,954$ & $14,341,167$ & $14,066,023$ & $13,798,315$ & $13,537,840$ & $13,284,401$ & $13,037,808$ & $12,797,875$ & $12,564,419$ & $12,337,267$ & $12,116,246$ & $11,901,190$ \\
\hline LONG TERM DEBT & - & - & - & - & - & - & - & - & - & - & - & - & - \\
\hline \multicolumn{14}{|l|}{ STOCKHOLDERS' EQUITY } \\
\hline Class A Common Stock (268,885 shares) & 100,000 & 100,000 & 100,000 & 100,000 & 100,000 & 100,000 & 100,000 & 100,000 & 100,000 & 100,000 & 100,000 & 100,000 & 100,000 \\
\hline Additional Paid-In Capital & $2,400,000$ & $2,400,000$ & $2,400,000$ & $2,400,000$ & $2,400,000$ & $2,400,000$ & $2,400,000$ & $2,400,000$ & $2,400,000$ & $2,400,000$ & $2,400,000$ & $2,400,000$ & $2,400,000$ \\
\hline Retained Earnings & $16,475,586$ & $16,475,586$ & $16,891,352$ & $17,309,745$ & $17,731,055$ & $18,155,574$ & $18,583,601$ & $19,015,441$ & $19,451,404$ & $19,891,807$ & $20,341,971$ & $20,797,227$ & $21,257,910$ \\
\hline Less: Treasury Stock & $(500,000)$ & $(500,000)$ & $(500,000)$ & $(500,000)$ & $(500,000)$ & $(500,000)$ & $(500,000)$ & $(500,000)$ & $(500,000)$ & $(500,000)$ & $(500,000)$ & $(500,000)$ & $(500,000)$ \\
\hline TOTAL EQUITY & $18,475,586$ & $18,475,586$ & $18,891,352$ & $19,309,745$ & $19,731,055$ & $20,155,574$ & $20,583,601$ & $21,015,441$ & $21,451,404$ & $21,891,807$ & $22,341,971$ & $22,797,227$ & $23,257,910$ \\
\hline TOTAL LIABILITIES \& CAPITAL & $33,099,540$ & $33,099,540$ & $33,232,519$ & $33,375,769$ & $33,529,370$ & $33,693,414$ & $33,868,003$ & $34,053,249$ & $34,249,279$ & $34,456,226$ & $34,679,238$ & $34,913,473$ & $35,159,100$ \\
\hline
\end{tabular}




\section{Appendix 2: 2006 Financial Statements}

\begin{tabular}{|c|c|c|c|c|c|c|c|c|c|c|c|c|c|}
\hline 2006 Income Statement & $12 / 31 / 06$ & Dec-06 & Nov-06 & Oct-06 & Sep-06 & Aug-06 & Jul-06 & Jun-06 & May-06 & Apr-06 & Mar-06 & Feb-06 & Jan-06 \\
\hline & Consolidated & & & & & & & & & & & & \\
\hline \multicolumn{14}{|l|}{ SALES } \\
\hline Gross Sales & $116,838,105$ & $16,442,912$ & $8,400,907$ & $8,359,112$ & $12,217,524$ & $8,276,143$ & $8,234,968$ & $12,793,998$ & $8,153,232$ & $8,112,669$ & $9,822,307$ & $8,032,147$ & $7,992,186$ \\
\hline Discounts & $(3,505,143)$ & $(493,287)$ & $(252,027)$ & $(250,773)$ & $(366,526)$ & $(248,284)$ & $(247,049)$ & $(383,820)$ & $(244,597)$ & $(243,380)$ & $(294,669)$ & $(240,964)$ & $(239,766$ \\
\hline Returns \& Allowances & $(1,971,762)$ & $(168,858)$ & $(168,018)$ & $(167,182)$ & $(166,350)$ & $(165,523)$ & $(164,699)$ & $(163,880)$ & $(163,065)$ & $(162,253)$ & $(161,446)$ & $(160,643)$ & $(159,844$ \\
\hline NET SALES & $111,361,200$ & $15,780,766$ & $7,980,862$ & $7,941,156$ & $11,684,648$ & $7,862,336$ & $7,823,220$ & $12,246,298$ & $7,745,571$ & $7,707,035$ & $9,366,192$ & $7,630,539$ & $7,592,576$ \\
\hline \multicolumn{14}{|l|}{ COST OF SALES } \\
\hline Standard Cost of Sales & $14,788,216$ & $1,266,437$ & $1,260,136$ & $1,253,867$ & $1,247,629$ & $1,241,421$ & $1,235,245$ & $1,229,100$ & $1,222,985$ & $1,216,900$ & $1,210,846$ & $1,204,822$ & $1,198,828$ \\
\hline Manufacturing Variances & 492,941 & 42,215 & 42,005 & 41,796 & 41,588 & 41,381 & 41,175 & 40,970 & 40,766 & 40,563 & 40,362 & 40,161 & 39,961 \\
\hline Total Cost of Sales & $15,281,156$ & $1,308,651$ & $1,302,141$ & $1,295,662$ & $1,289,216$ & $1,282,802$ & $1,276,420$ & $1,270,070$ & $1,263,751$ & $1,257,464$ & $1,251,208$ & $1,244,983$ & $1,238,789$ \\
\hline GROSS MANUFAC PROFIT & $96,080,044$ & $14,472,115$ & $6,678,721$ & $6,645,494$ & $10,395,432$ & $6,579,534$ & $6,546,800$ & $10,976,229$ & $6,481,820$ & $6,449,572$ & $8,114,984$ & $6,385,557$ & $6,353,788$ \\
\hline \multicolumn{14}{|l|}{ OPERATING EXPENSES } \\
\hline Engineering & $1,641,428$ & - & 168,018 & 167,182 & 4,875 & 165,523 & 164,699 & 163,880 & 163,065 & 162,253 & 161,446 & 160,643 & 159,844 \\
\hline Marketing & $2,050,817$ & - & 210,023 & 208,978 & 5,125 & 206,904 & 205,874 & 204,850 & 203,831 & 202,817 & 201,808 & 200,804 & 199,805 \\
\hline Salary Expenses & $10,605,276$ & 883,773 & 883,773 & 883,773 & 883,773 & 883,773 & 883,773 & 883,773 & 883,773 & 883,773 & 883,773 & 883,773 & 883,773 \\
\hline Depreciation Expense & 571,469 & 11,245 & 22,533 & 22,533 & 22,533 & 61,578 & 61,578 & 61,578 & 61,578 & 61,578 & 61,578 & 61,578 & 61,578 \\
\hline Total Operating Expenses & $14,868,990$ & 895,018 & $\begin{array}{l}1,284,347 \\
\end{array}$ & $1,282,466$ & 916,306 & 1,317,778 & $1,315,925$ & $\begin{array}{l}1,314,081 \\
\end{array}$ & 1,312,247 & $1,310,421$ & $1,308,605$ & $1,306,798$ & $1,305,000$ \\
\hline OPERATING PROFIT & $81,211,053$ & $13,577,097$ & $5,394,374$ & $5,363,028$ & $9,479,126$ & $5,261,756$ & $5,230,875$ & $9,662,148$ & $5,169,573$ & $5,139,151$ & $6,806,379$ & $5,078,759$ & $5,048,788$ \\
\hline \multicolumn{14}{|l|}{ NON-OPERATING INCOME } \\
\hline Interest Expense & $(197,176)$ & $(16,886)$ & $(16,802)$ & $(16,718)$ & $(16,635)$ & $(16,552)$ & $(16,470)$ & $(16,388)$ & $(16,306)$ & $(16,225)$ & $(16,145)$ & $(16,064)$ & $(15,984$ \\
\hline Other & $(49,294)$ & $(4,221)$ & $(4,200)$ & $(4,180)$ & $(4,159)$ & $(4,138)$ & $(4,117)$ & $(4,097)$ & $(4,077)$ & $(4,056)$ & $(4,036)$ & $(4,016)$ & $(3,996$ \\
\hline Total Non-Operating Inc/Exp & $(246,470)$ & $(21,107)$ & $(21,002)$ & $(20,898)$ & $(20,794)$ & $(20,690)$ & $(20,587)$ & $(20,485)$ & $(20,383)$ & $(20,282)$ & $(20,181)$ & $(20,080)$ & $(19,980$ \\
\hline PRETAX INCOME & $80,964,583$ & $13,555,989$ & $5,373,372$ & $5,342,130$ & $9,458,332$ & $5,241,066$ & $5,210,288$ & $9,641,663$ & $5,149,190$ & $5,118,869$ & $6,786,199$ & $5,058,678$ & $5,028,808$ \\
\hline PROVISION FOR INCOME TAXES & $31,576,187$ & $2,175,873$ & $2,162,910$ & $2,149,815$ & $2,136,976$ & $2,124,200$ & $2,111,485$ & $2,098,832$ & $2,086,240$ & $2,073,708$ & $2,061,237$ & $2,048,826$ & $2,036,475$ \\
\hline NET INCOME & $49,388,396$ & $11,380,116$ & $3,210,462$ & $3,192,315$ & $7,321,355$ & $3,116,866$ & $3,098,802$ & $7,542,831$ & $3,062,950$ & $3,045,161$ & $4,724,962$ & $3,009,853$ & $2,992,333$ \\
\hline
\end{tabular}




\begin{tabular}{|c|c|c|c|c|c|c|c|c|c|c|c|c|c|}
\hline 2006 Balance Sheet & $12 / 31 / 06$ & Dec-06 & Nov-06 & Oct-06 & Sep-06 & Aug-06 & Jul-06 & Jun-06 & May-06 & Apr-06 & Mar-06 & Feb-06 & Jan-06 \\
\hline & Consolidated & & & & & & & & & & & & \\
\hline CASH AND ST INVESTMENTS & $10,200,000$ & $10,200,000$ & $10,200,000$ & $10,200,000$ & $10,000,000$ & $10,000,000$ & $10,000,000$ & $10,000,000$ & $10,000,000$ & $10,000,000$ & $10,000,000$ & $10,000,000$ & $10,000,000$ \\
\hline \multicolumn{14}{|l|}{ ACCOUNTS RECEIVABLE } \\
\hline Gross Accounts Receivable & $12,688,582$ & $12,688,582$ & $1,680,181$ & $1,671,822$ & $4,663,505$ & $1,655,229$ & $1,646,994$ & $1,638,800$ & $1,630,646$ & $1,622,534$ & $1,614,461$ & $1,606,429$ & $1,598,437$ \\
\hline Reserve for Doubtful Accounts & $(168,858)$ & $(168,858)$ & $(168,018)$ & $(167,182)$ & $(166,350)$ & $(165,523)$ & $(164,699)$ & $(163,880)$ & $(163,065)$ & $(162,253)$ & $(161,446)$ & $(160,643)$ & $(159,844)$ \\
\hline Net Accounts Receivable & $12,519,724$ & $12,519,724$ & $1,512,163$ & $1,504,640$ & $4,497,154$ & $1,489,706$ & $1,482,294$ & $1,474,920$ & $1,467,582$ & $1,460,280$ & $1,453,015$ & $1,445,786$ & $1,438,593$ \\
\hline INVENTORIES & $5,947,255$ & $5,947,255$ & $6,007,329$ & $6,068,009$ & $6,129,302$ & $6,191,214$ & $6,253,751$ & $6,316,921$ & $6,380,728$ & $6,445,180$ & $6,510,283$ & $6,576,043$ & $6,642,468$ \\
\hline OTHER CURRENT ASSETS & $2,645,000$ & $2,645,000$ & $2,265,000$ & $2,265,000$ & $2,265,000$ & $1,900,000$ & $1,900,000$ & $1,800,000$ & $1,700,000$ & $1,700,000$ & $1,700,000$ & $2,500,000$ & $2,300,000$ \\
\hline TOTAL CURRENT ASSETS & $31,311,980$ & $31,311,980$ & $19,984,492$ & $20,037,649$ & $22,891,456$ & $19,580,920$ & $19,636,046$ & $19,591,840$ & $19,548,310$ & $19,605,460$ & $19,663,298$ & $20,521,829$ & $20,381,061$ \\
\hline \multicolumn{14}{|l|}{ FIXED ASSETS } \\
\hline Land and Buildings & $8,750,000$ & $8,750,000$ & $8,766,667$ & $8,783,333$ & $8,800,000$ & $8,816,667$ & $8,833,333$ & $8,850,000$ & $8,866,667$ & $8,883,333$ & $8,900,000$ & $8,916,667$ & $8,933,333$ \\
\hline Machinery and Equipment & $1,450,000$ & $1,450,000$ & $1,458,333$ & $1,466,667$ & $1,475,000$ & $1,483,333$ & $1,491,667$ & $1,500,000$ & $1,508,333$ & $1,516,667$ & $1,525,000$ & $1,533,333$ & $1,541,667$ \\
\hline \multicolumn{14}{|l|}{ Construction in Progress } \\
\hline Accumulated Depreciation & $1,614,080$ & $1,614,080$ & $1,596,257$ & $1,567,724$ & $1,539,191$ & $1,510,658$ & $1,443,079$ & $1,375,501$ & $1,308,923$ & $1,242,345$ & $1,175,767$ & $1,109,189$ & $1,042,610$ \\
\hline Net Fixed Assets & $11,814,080$ & $11,814,080$ & $11,821,257$ & $11,817,724$ & $11,814,191$ & $11,810,658$ & $11,768,079$ & $11,725,501$ & $11,683,923$ & $11,642,345$ & $11,600,767$ & $11,559,189$ & $11,517,610$ \\
\hline OTHER ASSETS & 875,000 & 875,000 & 875,000 & 875,000 & 875,000 & 875,000 & 875,000 & 875,000 & 875,000 & 875,000 & 875,000 & 875,000 & 875,000 \\
\hline TOTAL ASSETS & $44,001,059$ & $44,001,059$ & $32,680,748$ & $32,730,372$ & $35,580,647$ & $32,266,577$ & $32,279,125$ & $32,192,341$ & $32,107,233$ & $32,122,805$ & $32,139,065$ & $32,956,018$ & $32,773,671$ \\
\hline \multicolumn{14}{|l|}{ CURRENT LIABILITIES } \\
\hline Accounts Payable & $6,200,000$ & $6,200,000$ & $6,200,000$ & $6,200,000$ & $6,200,000$ & $6,200,000$ & $6,200,000$ & $6,200,000$ & $6,200,000$ & $6,200,000$ & $6,200,000$ & $6,200,000$ & $6,200,000$ \\
\hline Accrued Expenses & 46,486 & 46,486 & 46,026 & 45,570 & 45,119 & 935,761 & 926,496 & 917,323 & $2,393,389$ & $2,369,692$ & $2,346,230$ & $2,323,000$ & $2,300,000$ \\
\hline Unearned Revenue & 92,477 & 92,477 & 91,561 & 90,654 & 89,757 & 88,868 & 87,988 & 87,117 & $3,155,561$ & $3,124,318$ & $3,093,384$ & $4,819,288$ & $4,795,311$ \\
\hline Accrued Compensation & $2,400,000$ & $2,400,000$ & $2,400,000$ & $2,400,000$ & $2,400,000$ & $2,400,000$ & $2,400,000$ & $2,400,000$ & $2,400,000$ & $2,400,000$ & $2,400,000$ & $2,400,000$ & $2,400,000$ \\
\hline TOTAL CURRENT LIABILITIES & $8,738,963$ & $8,738,963$ & $8,737,587$ & $8,736,224$ & $8,734,876$ & $9,624,629$ & $9,614,485$ & $9,604,440$ & $14,148,951$ & $14,094,011$ & $14,039,614$ & $15,742,288$ & $15,695,311$ \\
\hline LONG TERM DEBT & - & - & - & - & - & - & - & - & - & - & - & - & - \\
\hline \multicolumn{14}{|l|}{ STOCKHOLDERS' EQUITY } \\
\hline Class A Common Stock ( 268,885 shares) & 100,000 & 100,000 & 100,000 & 100,000 & 100,000 & 100,000 & 100,000 & 100,000 & 100,000 & 100,000 & 100,000 & 100,000 & 100,000 \\
\hline Additional Paid-In Capital & $2,400,000$ & $2,400,000$ & $2,400,000$ & $2,400,000$ & $2,400,000$ & $2,400,000$ & $2,400,000$ & $2,400,000$ & $2,400,000$ & $2,400,000$ & $2,400,000$ & $2,400,000$ & $2,400,000$ \\
\hline Retained Earnings & $33,262,096$ & $33,262,096$ & $21,943,162$ & $21,994,148$ & $24,845,771$ & $20,641,948$ & $20,664,641$ & $20,587,901$ & $15,958,282$ & $16,028,794$ & $16,099,450$ & $15,213,730$ & $15,078,360$ \\
\hline Less: Treasury Stock & $(500,000)$ & $(500,000)$ & $(500,000)$ & $(500,000)$ & $(500,000)$ & $(500,000)$ & $(500,000)$ & $(500,000)$ & $(500,000)$ & $(500,000)$ & $(500,000)$ & $(500,000)$ & $(500,000)$ \\
\hline TOTAL EQUITY & $35,262,096$ & $35,262,096$ & $23,943,162$ & $23,994,148$ & $26,845,771$ & $22,641,948$ & $22,664,641$ & $22,587,901$ & $17,958,282$ & $18,028,794$ & $18,099,450$ & $17,213,730$ & $17,078,360$ \\
\hline TOTAL LIABILITIES \& CAPITAL & $44,001,059$ & $44,001,059$ & $32,680,748$ & $32,730,372$ & $35,580,647$ & $32,266,577$ & $32,279,125$ & $32,192,341$ & $32,107,233$ & $32,122,805$ & $32,139,065$ & $32,956,018$ & $32,773,671$ \\
\hline
\end{tabular}




\section{Appendix 3: 2006 Payroll Register}

\section{Salary Payroll Register}

\begin{tabular}{|c|c|c|c|c|c|}
\hline SSN & Name & Sex & DOB & DOE & Address \\
\hline $556-27-5184$ & Hollywood, L. & $\mathrm{F}$ & $5 / 21 / 1997$ & 4/30/1990 & 100 Plum St \\
\hline $180-56-8467$ & Watts, M. & $\mathrm{F}$ & $3 / 29 / 1971$ & $10 / 6 / 1997$ & 104 Iverson Lane \\
\hline $200-62-7967$ & Reagan, C. & $\mathrm{M}$ & $12 / 28 / 1970$ & $6 / 27 / 1988$ & 198 Anderson \\
\hline $183-66-7282$ & Dooley, D. & M & $7 / 14 / 1970$ & 6/7/1993 & 14 Anderson Ave \\
\hline 184-54-0080 & Livinghouse, L. & $\mathrm{M}$ & 9/23/1969 & 4/2/1990 & 198 Mayfield Dr \\
\hline $168-42-5854$ & Knechtle, D. & $\mathrm{F}$ & 3/19/1966 & $8 / 24 / 1998$ & 234 Apple Stree \\
\hline $210-56-0067$ & Knouse, L. & $\mathrm{F}$ & $6 / 6 / 1965$ & $9 / 22 / 1986$ & 1005 Compton Drive \\
\hline 261-89-7094 & Begone, $\mathrm{C}$. & M & $5 / 21 / 1965$ & 2/3/1997 & 747 Prairie Street \\
\hline $166-46-9328$ & Ostroski, J. & $\mathrm{F}$ & $10 / 16 / 1964$ & 9/19/1990 & 12 Harold Lane \\
\hline 203-56-3178 & Roth, B. & M & 4/7/1964 & $7 / 31 / 1989$ & 102 Willow Street \\
\hline 135-64-0109 & Hafer, F. & M & 2/20/1964 & $10 / 5 / 1987$ & 1049 Andrea Place \\
\hline $175-56-5365$ & Wolfe, K. & M & $1 / 13 / 1963$ & $5 / 2 / 1988$ & 104 Broad Street \\
\hline $199-52-6686$ & Zillhardt, M. & $\mathrm{F}$ & $10 / 14 / 1962$ & $11 / 5 / 1981$ & 19 Carpenter Blvd \\
\hline $182-58-2751$ & Hadley, R. & M & $1 / 31 / 1962$ & $5 / 13 / 1985$ & 98 Randolph \\
\hline 183-56-6884 & Borneman, M. & M & 10/26/1961 & 2/23/1998 & 934 Pharmore Drive \\
\hline $164-52-5332$ & Feick, S. & $\mathrm{F}$ & $7 / 27 / 1960$ & $11 / 2 / 1992$ & 51 Drexel Road \\
\hline $124-23-2342$ & Jones, $\mathrm{M}$ & M & $12 / 20 / 1959$ & $5 / 27 / 1986$ & 101 Waldorf Way \\
\hline 200-79-1113 & Nellson, $\mathrm{M}$ & M & $10 / 3 / 1959$ & 6/8/1997 & P.O Box 197 \\
\hline 101-01-0101 & Nelsen, M & $\mathrm{M}$ & $10 / 1 / 1959$ & 6/6/1997 & 123 Main Street \\
\hline $987-65-4321$ & Ostrrowski, J & $\mathrm{F}$ & 9/30/1959 & 6/5/1997 & 430 Orange \\
\hline $323-45-6789$ & Nelse, M & M & 9/29/1959 & 6/4/1997 & P.O Box 197 \\
\hline $204-56-4497$ & Marchione, T. & $\mathrm{F}$ & $8 / 3 / 1959$ & $9 / 26 / 1983$ & 189 Plate Ave \\
\hline 299-58-4277 & Bennett, J. & $\mathrm{M}$ & $5 / 8 / 1958$ & $1 / 6 / 1986$ & 280 Belmont Ave \\
\hline $166-46-9263$ & Haldeman, G. & $\mathrm{M}$ & 10/15/1957 & $10 / 11 / 1988$ & 1413 Appier Street \\
\hline $190-46-6702$ & Nelson, M. & M & $4 / 24 / 1957$ & $4 / 18 / 1983$ & P.O Box 197 \\
\hline $182-50-6491$ & Rohrbach, C. & $\mathrm{F}$ & $1 / 23 / 1957$ & $11 / 4 / 1991$ & $105 \mathrm{Mt}$, Mutumbo Circle \\
\hline $246-02-4513$ & Aldridge, $\mathrm{S}$. & M & $12 / 2 / 1955$ & $11 / 7 / 1994$ & 100 Bonner Blvd \\
\hline 219-64-3286 & Szurgot, Z. & $\mathrm{F}$ & $10 / 31 / 1952$ & 4/11/1994 & 1049 Andrea Place \\
\hline 200-38-2419 & Osmond, E. & $\mathrm{M}$ & $3 / 17 / 1948$ & 2/2/1998 & 234 Ferret Street \\
\hline $027-38-8275$ & Clarkson, R. E. & M & $2 / 8 / 1948$ & $7 / 24 / 1991$ & 5 Glen Loch \\
\hline $200-38-8317$ & Goodwin, V. & $\mathrm{F}$ & $10 / 13 / 1947$ & $11 / 4 / 1991$ & 109 Happy Valley Ave \\
\hline 241-78-1207 & Cagle, J. & $\mathrm{F}$ & $7 / 31 / 1947$ & $8 / 5 / 1985$ & 133 Bergdoll Terrace \\
\hline 200-38-2419 & Weicicoskie, C. & $\mathrm{M}$ & $10 / 22 / 1945$ & 2/13/1989 & 180 Hope Street \\
\hline $187-34-9193$ & Franklin, B. & M & $6 / 26 / 1945$ & 9/25/1967 & 998 Golf Road \\
\hline $279-40-0755$ & Yehl, R. & $\mathrm{F}$ & $12 / 14 / 1944$ & $1 / 13 / 1986$ & 1943 Pearson Place \\
\hline $171-36-7901$ & Stealing, I.M. & $\mathrm{F}$ & $10 / 13 / 1944$ & $11 / 11 / 1996$ & 15 Birch Tree Lane \\
\hline 203-34-3852 & Freeman, B. & $\mathrm{F}$ & $4 / 30 / 1944$ & $3 / 10 / 1969$ & 109 Marchant Manor \\
\hline $173-34-8850$ & Rios, A. & $\mathrm{F}$ & $8 / 17 / 1943$ & 2/9/1983 & 225 Ruby St \\
\hline $173-34-9496$ & Morganti, A. & $\mathrm{M}$ & $7 / 29 / 1942$ & $2 / 2 / 1965$ & 110 Columbus Ave \\
\hline $195-34-2650$ & Stamm, D. & M & $6 / 25 / 1942$ & $8 / 28 / 1961$ & 92 Maple St \\
\hline 203-30-0163 & Raymond, L. & M & $7 / 28 / 1939$ & $10 / 17 / 1966$ & 198 Cobbs Creek \\
\hline
\end{tabular}

\begin{tabular}{|c|c|c|c|c|c|c|}
\hline City & State & Zip & Annual & Bi-weekly & $\begin{array}{c}\text { Employer } \\
\text { Withholdings }\end{array}$ & $\begin{array}{l}\text { Individual } \\
\text { Withholdings }\end{array}$ \\
\hline Norristown & PA & 19402 & $35,330.00$ & $1,358.85$ & $6,253.41$ & $3,533.00$ \\
\hline Drexel Hill & PA & 19155 & $28,380.00$ & $1,091.54$ & $5,023.26$ & $2,838.00$ \\
\hline Overbrook & PA & 18320 & $1,256,325.00$ & $48,320.19$ & $222,369.53$ & $125,632.50$ \\
\hline Overbrook & PA & 19402 & $41,601.00$ & $1,600.04$ & $7,363.38$ & $4,160.10$ \\
\hline Philadelphia & PA & 19104 & $138,927.00$ & $5,343.35$ & $24,590.08$ & $13,892.70$ \\
\hline West Chester & PA & 19402 & $566,065.00$ & $21,771.73$ & $100,193.51$ & $56,606.50$ \\
\hline Overbrook & PA & 19402 & $32,857.00$ & $1,263.73$ & $5,815.69$ & $3,285.70$ \\
\hline Philadelphia & PA & 19402 & $14,873.00$ & 572.04 & $2,632.52$ & $1,487.30$ \\
\hline West Chester & PA & 19103 & $43,808.94$ & $1,684.96$ & $7,754.18$ & $4,380.89$ \\
\hline Mt. Laurel & NJ & 19402 & $58,878.00$ & $2,264.54$ & $10,421.41$ & $5,887.80$ \\
\hline Malvern & PA & 18320 & $69,581.00$ & $2,676.19$ & $12,315.84$ & $6,958.10$ \\
\hline Overbrook & PA & 19402 & $250,161.00$ & $9,621.58$ & $44,278.50$ & $25,016.10$ \\
\hline West Chester & NJ & 19103 & $212,804.00$ & $8,184.77$ & $37,666.31$ & $21,280.40$ \\
\hline Philadelphia & PA & 19103 & $2,334,106.00$ & $89,773.31$ & $413,136.76$ & $233,410.60$ \\
\hline Moorsetown & NJ & 19103 & $33,481.00$ & $1,287.73$ & $5,926.14$ & $3,348.10$ \\
\hline Norristown & PA & 19103 & $29,489.00$ & $1,134.19$ & $5,219.55$ & $2,948.90$ \\
\hline Philadelphia & $\mathrm{Pa}$ & 19103 & $130,000.00$ & $5,000.00$ & $23,010.00$ & $13,000.00$ \\
\hline West Chester & $\mathrm{PA}$ & 19382 & $96,740.00$ & $3,720.77$ & $17,122.98$ & - \\
\hline West Chester & PA & 19406 & $64,740.00$ & $2,490.00$ & $11,458.98$ & $6,474.00$ \\
\hline Malvern & PA & 19406 & $88,920.00$ & $3,420.00$ & $15,738.84$ & $8,892.00$ \\
\hline West Chester & PA & 19382 & $42,640.00$ & $1,640.00$ & $7,547.28$ & - \\
\hline Mt. Laurel & NJ & 19155 & $231,110.00$ & $8,888.85$ & $40,906.47$ & $23,111.00$ \\
\hline Philadelphia & PA & 18320 & $89,070.00$ & $3,425.77$ & $15,765.39$ & $8,907.00$ \\
\hline Norristown & PA & 18320 & $38,796.00$ & $1,492.15$ & $6,866.89$ & $3,879.60$ \\
\hline West Chester & PA & 19382 & $341,032.00$ & $13,116.62$ & $60,362.66$ & - \\
\hline Malvern & PA & 18320 & $36,393.00$ & $1,399.73$ & $6,441.56$ & $3,639.30$ \\
\hline Philadelphia & $\mathrm{PA}$ & 19103 & $33,843.00$ & $1,301.65$ & $5,990.21$ & $3,384.30$ \\
\hline Malvern & PA & 18320 & $69,581.00$ & $2,676.19$ & $12,315.84$ & $6,958.10$ \\
\hline Drexel Hill & PA & 19155 & $43,770.00$ & $1,683.46$ & $7,747.29$ & $4,377.00$ \\
\hline Wilmington & $\mathrm{DE}$ & 19402 & $532,908.00$ & $20,496.46$ & $94,324.72$ & $53,290.80$ \\
\hline Overbrook & $\mathrm{PA}$ & 19155 & $39,099.00$ & $1,503.81$ & $6,920.52$ & $3,909.90$ \\
\hline Norristown & PA & 19104 & $36,708.00$ & $1,411.85$ & $6,497.32$ & $3,670.80$ \\
\hline Mt. Laurel & NJ & 19104 & $42,692.00$ & $1,642.00$ & $7,556.48$ & $4,269.20$ \\
\hline Malvern & PA & 19155 & $41,277.00$ & $1,587.58$ & $7,306.03$ & $4,127.70$ \\
\hline Norristown & PA & 18320 & $35,360.00$ & $1,360.00$ & $6,258.72$ & $3,536.00$ \\
\hline Moorsetown & NJ & 19104 & $31,458.00$ & $1,209.92$ & $5,568.07$ & $3,145.80$ \\
\hline Drexel Hill & PA & 19103 & $48,249.00$ & $1,855.73$ & $8,540.07$ & $4,824.90$ \\
\hline Drexel Hill & PA & 19155 & $41,862.00$ & $1,610.08$ & $7,409.57$ & $4,186.20$ \\
\hline Overbrook & PA & 19103 & $54,613.00$ & $2,100.50$ & $9,666.50$ & $5,461.30$ \\
\hline & PA & 19104 & $50,061.00$ & $1,925.42$ & $8,860.80$ & $5,006.10$ \\
\hline \multirow[t]{2}{*}{ Drexel Hill } & $\mathrm{PA}$ & 19155 & $2,333,909.00$ & $89,765.73$ & $413,101.89$ & $233,390.90$ \\
\hline & & Total & $9,706,167.94$ & $373,314.15$ & $1,717,991.73$ & $922,575.59$ \\
\hline
\end{tabular}




\section{Appendix 4: Accounts Receivable Detailed Aging Report 12/31/06}

\begin{tabular}{|c|c|c|c|c|c|c|c|}
\hline \multicolumn{2}{|c|}{ As of December 31, 2006} & \multicolumn{6}{|c|}{ Accounts Receivable Detailed Aging } \\
\hline Customer \# & Customer Name & Customer Address & 0-30 Days & 30-60 Days & 60-90 Days & Over 90 Days & $\underline{\text { Customer Total }}$ \\
\hline \multirow[t]{5}{*}{101} & AAA Electronics & 111 Atlantic Blvd. Philadelphia, PA 19107 & & & & & \\
\hline & & Inv. \# 350 & & 10,850 & & & \\
\hline & & Inv. \# 370 & & 82,700 & & & \\
\hline & & Inv. \# 410 & 6,800 & & & & \\
\hline & & & & & & & 100,350 \\
\hline \multirow[t]{4}{*}{102} & Abba, Inc. & 350 Pine Rd. Baltimore, MD 21201 & & & & & \\
\hline & & Inv. \# 220 & & & & 170,370 & \\
\hline & & Inv. \# 360 & & 26,395 & & & \\
\hline & & & & & & & 196,765 \\
\hline \multirow[t]{4}{*}{103} & Anderson, Inc. & 400 West St. San Jose, CA 95112 & & & & & \\
\hline & & Inv. \# 100 & & & & 11,200 & \\
\hline & & Inv. \# 540 & 6,023 & & & & \\
\hline & & & & & & & 17,223 \\
\hline \multirow[t]{5}{*}{104} & Boomin Systems Co. & 890 Broad St. Philadelphia, PA 19107 & & & & & \\
\hline & & Inv. \# 150 & & & & 33,350 & \\
\hline & & Inv. \# 390 & & 6,954 & & & \\
\hline & & Inv. \# 420 & 3,350 & & & & \\
\hline & & & & & & & 43,654 \\
\hline \multirow[t]{3}{*}{106} & Better Quality Stereos & 350 Market St. Philadelphia, PA 19106 & & & & & \\
\hline & & Inv. \# 380 & & 3,030 & & & \\
\hline & & & & & & & 3,030 \\
\hline \multirow{4}{*}{105} & Big Buy Video Center & 100 Park Ave. New York, NY 10001 & & & & & \\
\hline & & Inv. \# 180 & & & & 12,030 & \\
\hline & & Inv. \# 210 & & 8,979 & & & \\
\hline & & & & & & & 21,009 \\
\hline \multirow[t]{4}{*}{107} & Circuit Audio and Video & 240 Mall Blvd. King of Prussia, PA 19406 & & & & & \\
\hline & & Inv. \# 110 & & & & 187,150 & \\
\hline & & Inv. \# 120 & & & & 137,040 & \\
\hline & & & & & & & 324,190 \\
\hline \multirow[t]{3}{*}{108} & Cool Sounds, Inc. & 620 Cool Ave. Philadelphia, PA 19107 & & & & & \\
\hline & & Inv. \# 130 & & & 41,600 & & \\
\hline & & & & & & & 41,600 \\
\hline 109 & Nelson's Stereo Supply & 123 Main St. King of Prussia, PA 19406 & & & & & \\
\hline & & Inv. \# 230 & & & 5,076 & & \\
\hline & & Inv. \# 250 & & & 379 & & \\
\hline & & Inv. \# 340 & & & & 28,675 & \\
\hline & & & & & & & 34,130 \\
\hline 110 & Def Systems, Inc. & 180 Front St. Philadelphia, PA 19106 & & & & & \\
\hline & & Inv. \# 140 & & & 9,203 & & \\
\hline & & Inv. \# 240 & & & 7,700 & & \\
\hline & & & & & & & 16,903 \\
\hline 111 & Electronics-R-Us & 230 Infringement St. Philadelphia, PA 19107 & & & & & \\
\hline & & Inv. \# 160 & & & & 8,506 & \\
\hline & & & & & & & 8,506 \\
\hline 112 & Fast Eddie's & 870 Quick Blvd. Philadelphia, PA 19107 & & & & & \\
\hline & & Inv. \# 260 & & & & 9,900 & \\
\hline & & Inv. \# 310 & & & & 2,010 & \\
\hline & & Inv. \# 320 & & & & 1,902 & \\
\hline & & & & & & & 13,812 \\
\hline 113 & General Video Supply & 100 GVS St. New York , NY 10001 & & & & & \\
\hline & & Inv. \# 170 & & & & 12,660 & \\
\hline & & Inv. \# 330 & 10,950 & & & & \\
\hline & & & & & & & 23,610 \\
\hline 114 & Granny's Stereos & 900 Old St. Philadelphia, PA 19107 & & & & & \\
\hline & & Inv. \# 460 & 30,501 & & & & \\
\hline & & Inv. \# 670 & 314,339 & & & & \\
\hline & & & & & & & 344,840 \\
\hline 115 & Kenson Co. & 430 Anderson Ave. New York, NY 10001 & & & & & \\
\hline & & Inv. \# 470 & 5,900 & & & & \\
\hline & & Inv. \# 680 & & & & 187,734 & \\
\hline & & & & & & & 193,634 \\
\hline 116 & Nelsons \# 1 Electronics & 123 Main St. King of Prussia, PA 19406 & & & & & \\
\hline & & Inv. \# 115 & & 16,050 & & & \\
\hline & & Inv. \# 175 & & & & 32,700 & \\
\hline & & & & & & & 48,750 \\
\hline 117 & Phat Stereo Systems, Inc. & 320 Money St. Philadelphia, PA 19107 & & & & & \\
\hline & & Inv. \# 480 & & 3,030 & & & \\
\hline & & Inv. \# 630 & 2,018 & & & & \\
\hline & & Inv. \# 640 & & & 3,400 & & \\
\hline
\end{tabular}




\begin{tabular}{|c|c|c|c|c|c|c|c|}
\hline \multicolumn{8}{|c|}{ As of December 31, 2006} \\
\hline Customer \# & Customer Name & Customer Address & 0-30 Days & 30-60 Days & 60-90 Days & Over 90 Days & Customer Total \\
\hline \multirow[t]{4}{*}{118} & Pilot Electronics & 500 System Ave. San Jose, CA 95112 & & & & & \\
\hline & & Inv. \# 700 & & & & 36,530 & \\
\hline & & Inv. \# 720 & & & & 46,017 & \\
\hline & & & & & & & 82,547 \\
\hline \multirow[t]{4}{*}{119} & Stereos Inc. & 100 Sony St. San Jose, CA 95112 & & & & & \\
\hline & & Inv. \# 490 & 17,250 & & & & \\
\hline & & Inv. \# 650 & 3,489 & & & & \\
\hline & & & & & & & 20,739 \\
\hline \multirow[t]{3}{*}{120} & Swifty's Stereo Outlet & 200 Sutton Ave. Philadelphia, PA 19107 & & & & & \\
\hline & & Inv. \# 520 & & 6,210 & & & \\
\hline & & & & & & & 6,210 \\
\hline \multirow[t]{4}{*}{121} & Joe's Electronics & 12 Main Street & & & & & \\
\hline & & Inv. \# 699 & 26,750 & & & & \\
\hline & & Inv. \# 339 & 2,500 & & & & \\
\hline & & Inv. \# 219 & & & 24,200 & & \\
\hline \multirow[t]{4}{*}{122} & Too Loud Electronics & 460 Def Ave. Phialdelphia, PA 19106 & & & & & 53,450 \\
\hline & & Inv. \# 450 & & 6,500 & & & \\
\hline & & Inv. \# 620 & & 3,490 & & & \\
\hline & & & & & & & 9,990 \\
\hline \multirow[t]{4}{*}{123} & U Need Electronics & 110 S. High St. Philadelphia, PA 19107 & & & & & \\
\hline & & Inv. \# 500 & 47,333 & & & & \\
\hline & & & & & & & 47,333 \\
\hline & & 700 Trailer Blvd. Philadelphia, PA & & & & & \\
\hline \multirow[t]{5}{*}{124} & WT Electronics & 19106 & & & & & \\
\hline & & Inv. \# 190 & & & & 4,875 & \\
\hline & & Inv. \# 430 & & & 22,986 & & \\
\hline & & & & & & & 27,861 \\
\hline & & Total & 477,202 & 174,188 & 114,544 & 922,648 & $1,688,582$ \\
\hline
\end{tabular}




\section{Appendix 5: Accounts Payable Aging Report 12/31/06 Accounts Payable Aging Report \\ As of December 31, 2006}

Vendor \# Vendor Name

Address

0-30 Days

30-60 Days

60-90 Days

Over 90 Days

$\underline{\text { Total Due Per Vendor }}$

Allison Wholesalers

112 Ridge Avenue, Phila, PA 19128

Inv \# 33456A
Inv. \# 45267A
Inv. \# 45645A

134,567

83,412

347,654

405 Hollywood Blvd., Los Angeles, CA 95178

Inv. \# 220

Inv. \# 360

34,532

23,456

123 Market St, Phila, PA 19134

Inv. \# 345678

Inv. \# 345870

45,634

345,621

45,634

208 Coffeehouse Systems 145 West Avenue, New York, NY 10023 Inv. \# 180
Inv. \# 210

45,345

23,452

DMX Wholesalers

432 Pine St., Phila, PA 19128

Inv. \# 380

$210 \quad$ EXS Systems

340 Norristown Drive, Wilmington, DE 19324

Inv. \# 2345

Inv. \# 2476

123,400

123,400

$211 \quad$ EZ PASS Electronics

344 41st Street, Avalon, NJ 08234

Inv. \#130

$212 \quad$ Fellicity GrandStand

143 West Main Blvd, New York, NY 10023

$$
\begin{aligned}
& \text { Inv. \# } 465 \\
& \text { Inv. \# } 487
\end{aligned}
$$

Inv. \# 521

234 Race Street, Philadelphia, PA 19106

Inv. \# 34A

Inv. \# 45A

54,321

54,321

34,543

124,564

34,543

293,650

$\begin{array}{ll}213 & \text { GPX } \\ 214 & \text { Nelsen Temp Agency } \\ 215 & \text { IDX }\end{array}$

PO Box 197, West Chester, PA 19382

Inv. \# 160

245 South Street, Philadelphia, PA 19107

$$
\begin{aligned}
& \text { Inv. \# } 260 \\
& \text { Inv. \# } 310 \\
& \text { Inv. \# } 320 \\
& \text { Inv. \# } 320
\end{aligned}
$$

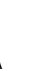

285,060

401,770

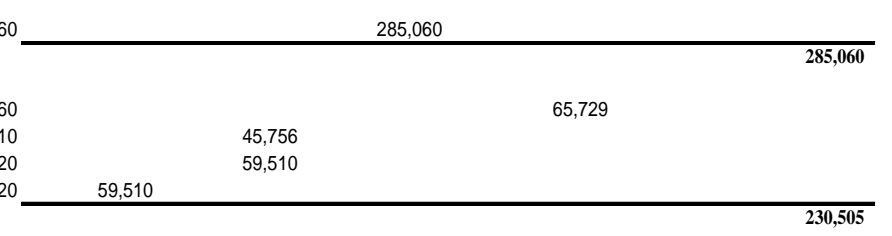

456 West 41st Street. New York, NY 10001

Inv. \# 170

126,600

Inv. \# 330

119,000

245,600

$217 \quad$ JR Nickel

900 Old St. Philadelphia, PA 19107

\begin{tabular}{lr} 
Inv. \# 563 & 110,020 \\
Inv. \# 894 & 140,500 \\
\hline
\end{tabular}

250,520

430 Anderson Ave. New York, NY 10001

\begin{tabular}{rr} 
Inv. \# 47023 & 47,133 \\
Inv. \# 47056 & 132,010 \\
\hline
\end{tabular}

MN Consulting

234 Market Street, Philadelphia, PA 19107

\begin{tabular}{lr} 
Inv. \# 115 & 123,450 \\
Inv. \# 175 & 34,532 \\
\hline
\end{tabular}

179,143 


\begin{tabular}{|c|c|c|c|c|c|c|c|}
\hline \multicolumn{8}{|c|}{ As of December 31, 2006} \\
\hline Vendor \# & Vendor Name & Address & 0-30 Days & 30-60 Days & 60-90 Days & Over 90 Days & $\underline{\text { Total Due Per Vendor }}$ \\
\hline \multirow[t]{5}{*}{205} & Nelson's Consulting & PO Box 197, West Chester, PA 19382 & & & & & \\
\hline & & Inv. \# 25 & & 56,432 & & & \\
\hline & & Inv. \# 26 & 14,234 & & & & \\
\hline & & Inv. \# 27 & 45,345 & & & & \\
\hline & & & & & & & 116,011 \\
\hline \multirow[t]{5}{*}{220} & Old School Limited & 2001 Market Street, Phila, PA 19128 & & & & & \\
\hline & & Inv. \# 5672 & & 30,300 & & & \\
\hline & & Inv. \# 5684 & 820,180 & & & & \\
\hline & & Inv. \# 5573 & & & 134,000 & & \\
\hline & & & & & & & 984,480 \\
\hline \multirow[t]{4}{*}{223} & Pilot Electronics & 500 System Ave. San Jose, CA 95112 & & & & & \\
\hline & & Inv. \# 700 & 30,590 & & & & \\
\hline & & Inv. \# 720 & 54,969 & & & & \\
\hline & & & & & & & 85,559 \\
\hline \multirow[t]{4}{*}{224} & Stereos Inc. & 100 Sony St. San Jose, CA 95112 & & & & & \\
\hline & & Inv. \# 49002 & 145,000 & & & & \\
\hline & & Inv. \# 49008 & 134,890 & & & & \\
\hline & & & & & & & 279,890 \\
\hline \multirow[t]{3}{*}{229} & Storage Incorporated & 200 S. Broad St, Philadelphia, PA 19134 & & & & & \\
\hline & & Inv. \# 845 & & 162,100 & & & \\
\hline & & & & & & & 162,100 \\
\hline \multirow[t]{4}{*}{241} & Timmy's Electronics Shop & 430 Anderson Ave. New York, NY 10001 & & & & & \\
\hline & & Inv. \# 56445 & 47,151 & & & & \\
\hline & & Inv. \# 56876 & 132,010 & & & & \\
\hline & & & & & & & 179,161 \\
\hline \multirow[t]{4}{*}{242} & Tony's Wholesalers & 5676 Market Street, Philadelphia, PA 19107 & & & & & \\
\hline & & Inv. \# 450 & & 35,000 & & & \\
\hline & & Inv. \# 620 & & 534,900 & & & \\
\hline & & & & & & & 569,900 \\
\hline \multirow[t]{3}{*}{243} & XYZ Decision Stereos & 23 Main St, Wilmington, DE 19345 & & & & & \\
\hline & & Inv. \# 50034 & 69,994 & & & & \\
\hline & & & & & & & 69,994 \\
\hline \multirow[t]{5}{*}{244} & Y Ask For Anything More & 700 Main St, Phila, PA 19127 & & & & & \\
\hline & & Inv. \# 190420 & & & & 248,750 & \\
\hline & & Inv. \# 190378 & & 29,865 & & & \\
\hline & & & & & & & 278,615 \\
\hline & & Total Per Period & $2,731,572$ & $1,431,896$ & $1,067,273$ & 969,259 & $6,200,000$ \\
\hline
\end{tabular}


Journal of Business Case Studies - March 2008

Volume 4, Number 3

NOTES 